\title{
Additional Mid-Peripheral Biopsies to the Systematic Lateral-Peripheral Sextant Biopsy Provides an Improvement in Prostate Cancer Detection in Japanese Men
}

\author{
Maki Yoshioka, Hideo Sasaki, Miki Yoshiike, Shiari Nozawa, Wataru Usuba, \\ Kouichirou Aida, Nozomi Hayakawa, Ryuto Nakazawa, and Eiji Kikuchi
}

(Received for Publication: September 10, 2020)

\begin{abstract}
Purpose: The aims of this study were to evaluate 1) in transrectal biopsy, whether additional 4-core biopsies of the mid-peripheral region after lateral-peripheral sextant biopsy in the peripheral zone can improve the prostate cancer detection rate, and 2) the preventive role of flomoxef sodium and levofloxacin against infectious complications.

Material and Methods: We retrospectively analyzed the records of 1541 patients who underwent initial transrectal ultrasound guided prostate biopsies at our hospital between January 2003 and November 2016. Two hundred and fifty-one patients underwent sextant prostate biopsy and 1290 underwent 10-core biopsy. The cancer detection rates of these two methods were analyzed. Since April 2009, a single-dose of flomoxef sodium (500 $\mathrm{mg}$ ) has been injected immediately before the 10-core biopsy in addition to levofloxacin for antimicrobial prophylaxis. The infectious complication rate among biopsied patients with or without flomoxef sodium was also evaluated.

Results: The additional 4-cores biopsies significantly increased the cancer detection rate compared with sextant biopsy, especially in a subgroup of patients with a serum prostate specific antigen level of 4.0-10.0 ng/ml (38.5\% vs $28.7 \%, \mathrm{p}=0.032)$ without increasing the rate of clinically insignificant cancer $(5.2 \% \mathrm{vs} 7.5 \%$, $\mathrm{p}=0.367$ ). Moreover, the incidence rate of acute prostatitis in 10-core-biopsied patients who were administered flomoxef sodium in addition to levofloxacin was significantly lower than in those administered levofloxacin alone $(0.4 \%$ vs $4.6 \%$, p<0.0001).

Conclusions: The additional 4-core biopsies significantly increased the cancer detection rate compared with sextant biopsy without increasing the rate of clinically insignificant cancer. Moreover, flomoxef sodium together with levofloxacin may have preventive effects against infectious complications.
\end{abstract}

\section{Key words}

Prostate biopsy, prostate cancer, antibiotics, infectious complication

\section{Introduction}

The number of patients with prostate cancer in Japan is increasing, and the incidence rate of prostate cancer surpassed those of stomach and lung cancer in $2015^{1}$. To diagnose prostate cancer, ultrasoundguided random systematic prostate sextant biopsy has been used as the standard method for several deca- $\mathrm{des}^{2}$. To date, numerous biopsy strategies have been introduced, including extended sampling schemes ${ }^{3,4)}$. At present, evidenced-based clinical practice guidelines for prostate cancer (Japanese Urological Association, 2016 edition) recommend obtaining 10 to 12 biopsy samples, mainly in the peripheral zone, as an initial biopsy setting ${ }^{1)}$.

At our hospital, sextant biopsy cores were ini- 
tially obtained from the bilateral lateral apex, midgland, and base at the edge of the peripheral zone of the lateral peripheral region according to the method described by Terris et $\mathrm{al}^{5}$. Then, 10 -core prostate biopsy was introduced with additional 4-core biopsies obtained from the base and mid gland in the mid-peripheral region bilaterally. In general, however, it is thought that additional biopsies can increase the complication rate, especially prostatitis.

Antimicrobial prophylaxis is a useful strategy to prevent infectious complications. In the setting of transrectal prostate biopsy, antimicrobial prophylaxis is recommended by the Japanese guidelines for perioperative infection prevention in the field of urology6). Among infectious complications, although sepsis is a rare condition, if it develops after transrectal prostate biopsy, it can be serious and life threatening ${ }^{7}$. In our hospital, a single high dose of levofloxacin had been traditionally administered. Since 2009 , a single intravenous injection of flomoxef sodium $(500 \mathrm{mg}$ ) has been additionally administered immediately before the procedure to all patients. In the present study, we evaluated 1) whether additional 4-core biopsies of the mid-peripheral region to the lateral-peripheral region in the peripheral zone improves the prostate cancer (PCA) detection rate, and 2) the preventive role of flomoxef sodium and levofloxacin on the rate of infectious complications in the era of drug resistance.

\section{Materials and Methods}

\section{Patient selection}

Between January 2003 and November 2016, initial transrectal ultrasound guided systematic prostate needle biopsies were performed on 1541 patients for prostate cancer diagnosis at St. Marianna University Hospital. In this study, the cases of re-biopsy were excluded. Indications for biopsy were a serum prostate specific antigen (PSA) level $\geq 4.0 \mathrm{ng} / \mathrm{ml}$ and/or abnormal findings by digital rectal examination. No patients with a prior diagnosis of prostate cancer or hormonal manipulation were included. Patient ages ranged from 36 to 92 years (mean, 69.9 years).

\section{PSA assay and PSAD assessment}

Serum PSA was quantified by the Access ${ }^{\circledR} \mathrm{Hy}-$ britech ${ }^{\circledR}$ PSA Assay (Beckman Coulter, Inc., Brea. CA, USA) before May 7, 2009, and by the chemilumi ACS-ePSA® Assay (Siemens Healthcare, Tokyo, Japan) between May 8, 2009 and September 27, 2015. The Lumipulse Presto® PSA Assay (Fujirebio, Shinjuku, Japan) was used thereafter. PSA density
(PSAD) was calculated by dividing the total PSA level by the prostate volume (PV).

\section{The procedure of transrectal ultrasound-guided prostate needle biopsy}

Antiplatelet therapy and anticoagulant therapy were discontinued before biopsy. Oral antibiotics consisting of levofloxacin at $500 \mathrm{mg}$ were administered 3 days before biopsy and continued for 5 days. Since April 2009, single-dose intravenous injections of $500 \mathrm{mg}$ of flomoxef sodium have been administered immediately before the procedure. The biopsy was performed with patients in the lateral decubitus position. Following digital rectal examination, transrectal ultrasound was performed using $2 \%$ xylocaine gel. The prostate was scanned in the transverse and sagittal planes, and PV was calculated using the formula for a prolate ellipsoid (width $\times$ length $\times$ height $\times 0.52$ ). The width and height were calculated from the transverse ultrasonographic view at the greatest distance. The length was calculated from the sagittal plane by measuring the longest cephalad-to-cauda dimension. Systematic prostate biopsies were performed with spring loaded 18-gauge needles driven by the biopsy gun (Bard Magnum ${ }^{\circledR}$, Bard Medical, Covington, GA, USA) under transrectal ultrasound guidance.

Sextant biopsy with a total of 6 cores was obtained from the bilateral lateral apex, mid-gland, and base of the far lateral peripheral zone. For 10-core prostate biopsy, additional 4-core biopsy samples were obtained from the base and mid-gland in the mid-peripheral region bilaterally in the peripheral zone according to Hodge et al. (Figure 1) $)^{2}$. Prostate intraepithelial neoplasm or atypia was defined as no malignancy.

\section{Evaluation of clinical parameters}

We retrospectively reviewed the medical records to evaluate the clinical features including age, PSA, $\mathrm{PV}$, positive core number, detected cancer, Gleason score, and complications associated with prostate biopsy (bleeding, acute epididymitis, vasovagal response, acute prostatitis, and urinary retention). Bleeding was defined as that requiring some form of treatment such as additional endoscopic hemostasis or insertion of a gelatin sponge (SPONGOSTAN ${ }^{\mathrm{TM}}$ Anal, Ethicon, Inc., Johnson \& Johnson, New Brunswick, NJ, USA) into the rectum once after the procedure. All cases of acute epididymitis developing within 6 months of biopsy were included as compli- 
A: Transverse image

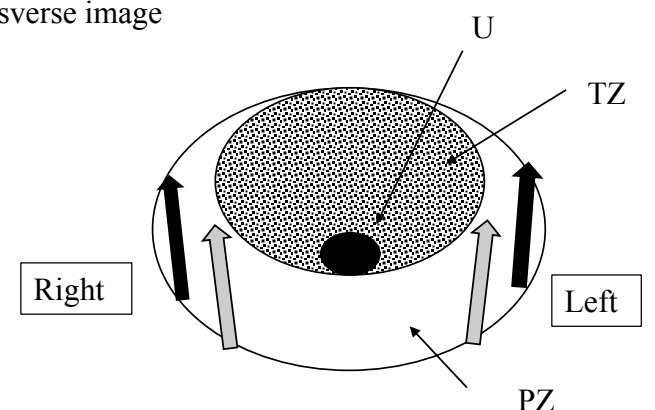

B: Sagittal image
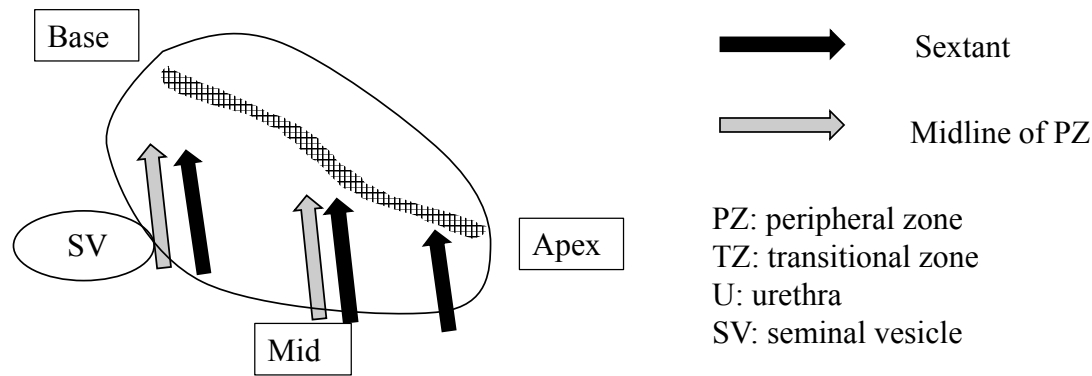

PZ: peripheral zone

TZ: transitional zone

U: urethra

SV: seminal vesicle

Figure 1. Schema of transrectal prostate biopsy. Sextant biopsy with a total of 6 cores was performed from the bilateral lateral apex, mid-gland, and base on the edge of the peripheral zone as the lateral-peripheral region (black arrow), and for 10-core prostate biopsy, additional 4-core biopsy samples were obtained from the base and mid-gland in the mid-peripheral region bilaterally in the peripheral zone (gray arrows). (A) Transverse image and (B) sagittal image. U, urethra; PZ, peripheral zone; SV. Seminal vesicle; TZ, transition zone.

cations attributed to the biopsy procedure ${ }^{8)}$. Vasovagal response was defined as bradycardia and hypotension during the biopsy. Acute prostatitis was defined as hospitalization for the first 30 days after biopsy due to urinary tract infection with fever (body temperature higher than $\left.38.5^{\circ} \mathrm{C}\right)^{9}$. The definition of sepsis was based on the 2001 International Sepsis Definitions Conference ${ }^{10)}$.

According to the 2016 Japanese Prostate Cancer Guidelines, the indications for active surveillance (AS) are a PSA of $10 \mathrm{ng} / \mathrm{ml}$ or less, PSAD of 0.2 or less, Gleason score of 6 or less, clinical stage T2 or less, and 2 or less cancer-positive cores ${ }^{1}$. We assumed patients with indications for AS to have clinically insignificant cancers.

\section{Statistical Analysis}

Continuous and categorical variables between the two groups (sextant and 10-core biopsy groups) were assessed by the t-test or Mann-Whitney $\mathrm{U}$ test and the $\chi^{2}$-test, respectively. A logistic regression analysis was used to identify a significant set of indicators associ- ated with the incidence of complications among all patients who underwent prostate biopsy. P-values $<0.05$ were considered significant. Statistical analyses were performed using JMP PRO ver.13 (SAS Institute, Cary, NC, USA). The study was approved by the St. Marianna University School of Medicine bioethics committee (approval number: 4146).

\section{Results}

\section{Patient background}

The characteristics of patients in the sextant biopsy and 10-core biopsy groups are summarized in Table 1. Of 1541 study subjects, 251 underwent sextant prostate biopsy (sextant biopsy group) and 1290 underwent 10-core biopsy (10-core biopsy group). Patient age and PSA value were significantly higher, and $\mathrm{PV}$ was significantly larger in the sextant biopsy group $(\mathrm{p}=0.0067,0.0067$, and $\mathrm{p}=0.0002$, respectively).

\section{Cancer detection rate between the sextant biopsy group and 10-core biopsy group}

Cancer was detected in 659 of 1290 patients 
$(51.0 \%)$ in the 10-core biopsy group and in 111 of 251 patients $(44.2 \%)$ in the sextant biopsy group, with a significant difference between the groups ( $\mathrm{p}=0.0047$ ). The cancer detection rates according to PSA, PSAD, and PV categories between the two groups are shown in Table 2. In a subgroup of patients with a PSA of $4.0-10.0 \mathrm{ng} / \mathrm{ml}$, the cancer detection rate in the 10-core biopsy group was $38.5 \%$, which was significantly higher than that in the sextant biopsy group (28.7\%, $\mathrm{p}=0.032)$. No significant differences in cancer detection rates were observed between 10-core biopsy and sextant biopsy groups in subgroups of patients with a PSA of 10.1-20.0 ng/ml, PSA of $>20 \mathrm{ng} / \mathrm{ml}$, PSAD of $<0.2$, PSAD of $\geq 0.2$, PV of $<20 \mathrm{ml}$, PV of $20-50 \mathrm{ml}$, or PV of $>50 \mathrm{ml}$.

The rate of patients with indications for AS (clinically insignificant cancer) was 7.5\% (7 of 93 patients) in the sextant biopsy group, which was not significantly different from that in the 10-core biopsy group $(5.2 \%, 29$ of 556 patients, $\mathrm{p}=0.367)$.

\section{Cancer regions detected by 10-core biopsy in prostate cancer patients}

Of 659 patients who underwent 10 -core biopsy and were found to have prostate cancer, $56(8.5 \%)$, $150(22.8 \%)$, and $453(68.7 \%)$ had cancer found in the mid-peripheral region only, lateral peripheral region only, and both regions, respectively. The distribution of patients who underwent 10-core biopsy and were found to have prostate cancer according to PSA, PSAD, and PV categories are shown in Table 3. According to the PSA level, cancer detection rates for the mid-peripheral region only in patients with a PSA of $4.0-10.0,10.1-20.0$, and $>20 \mathrm{ng} / \mathrm{ml}$ were $12.5 \%$, $8.0 \%$, and $3.4 \%$, respectively.

In 56 patients who underwent 10-core biopsy and in whom only the mid-peripheral region was detected, $38(67.8 \%), 17(30.4 \%), 1(1.8 \%)$, and zero patients had $1,2,3$, and 4 positive cores out of 4 , respectively. In addition, the highest total Gleason scores of $6,7,8$, and 9 were observed in $27(48.2 \%)$, $21(37.5 \%), 5(8.9 \%)$, and $3(5.4 \%)$ patients, respectively.

\section{Complication rate among biopsied patients with or without flomoxef sodium}

The overall complication rate in 10-core-biopsied patients who were administered levofloxacin alone

Table 1. Patient Background

\begin{tabular}{|c|c|c|c|c|c|c|c|}
\hline \multirow{2}{*}{ Parameters } & \multicolumn{3}{|c|}{ Sextant biopsy group } & \multicolumn{3}{|c|}{ 10-core biopsy group } & \multirow[b]{2}{*}{$\mathrm{p}$ value } \\
\hline & Mean \pm SD & Median & Range & Mean \pm SD & Median & Range & \\
\hline age (year)* & $70.8 \pm 7.4$ & 71 & $50-88$ & $69.4 \pm 7.8$ & 70 & $36-92$ & 0.0067 \\
\hline PSA (ng/ml)* & $74.6 \pm 407.0$ & 9.8 & $4.0-4670$ & $49.5 \pm 332.8$ & 8.6 & $4.0-5488$ & 0.0067 \\
\hline PSAD ** & $1.59 \pm 6.4$ & 0.259 & $0.05-74.2$ & $1.70 \pm 22.3$ & 0.285 & $0.04-711.6$ & 0.8223 \\
\hline $\mathrm{PV}(\mathrm{ml})^{* *}$ & $44.4 \pm 23.0$ & 40 & $10.5-162.8$ & $37.6 \pm 24.0$ & 31.1 & $11.3-214$ & 0.0002 \\
\hline
\end{tabular}

SD, standard deviation; PSA, prostate specific antigen; PSAD, PSA density; PV, prostate volume

* patient number, $\mathrm{n}=251$ in sextant biopsy group and $\mathrm{n}=1290$ in 10-core biopsy group

** patient number, $\mathrm{n}=213$ in sextant biopsy group and $\mathrm{n}=1106$ in 10-core biopsy group

Table 2. Cancer Detection Rates between Sextant Biopsy and 10-Core Biopsy Groups According to PSA, PSAD, and PV Categories

\begin{tabular}{|c|c|c|c|c|c|c|c|c|}
\hline & & \multicolumn{3}{|c|}{ Sextant biopsy group } & \multicolumn{3}{|c|}{10 -core biopsy group } & \multirow[b]{2}{*}{$\mathrm{p}$ value } \\
\hline & & $\begin{array}{c}\text { Patients with } \\
\text { cancer detected }\end{array}$ & Total patients & $\begin{array}{c}\text { Cancer detection } \\
\text { rate }(\%)\end{array}$ & $\begin{array}{c}\text { Patients with } \\
\text { cancer detected }\end{array}$ & Total patients & $\begin{array}{c}\text { Cancer detection } \\
\text { rate }(\%)\end{array}$ & \\
\hline \multirow{3}{*}{ PSA (ng/ml) } & $4.0-10.0$ & 37 & 129 & 28.7 & 289 & 750 & 38.5 & 0.032 \\
\hline & $10.1-20.0$ & 25 & 58 & 43.1 & 163 & 298 & 54.7 & 0.106 \\
\hline & $>20.0$ & 49 & 64 & 76.6 & 207 & 242 & 85.5 & 0.084 \\
\hline \multirow{2}{*}{ PSAD } & $<0.2$ & 15 & 80 & 18.8 & 91 & 353 & 25.8 & 0.187 \\
\hline & $\geq 0.2$ & 81 & 133 & 60.9 & 472 & 753 & 62.7 & 0.696 \\
\hline \multirow{3}{*}{$\mathrm{PV}(\mathrm{ml})$} & $<20$ & 12 & 17 & 70.6 & 139 & 213 & 65.3 & 0.656 \\
\hline & $20-50$ & 63 & 123 & 51.2 & 349 & 652 & 53.5 & 0.638 \\
\hline & $>50$ & 21 & 73 & 28.8 & 75 & 241 & 31.1 & 0.702 \\
\hline
\end{tabular}

PSA, prostate specific antigen; PSAD, PSA density; PV, prostate volume; N, number 
was $8.8 \%$, which was significantly higher than that in sextant-biopsied patients who were administered levofloxacin alone $(3.6 \%, \mathrm{p}=0.0099)$ and that in 10-corebiopsied patients who were administered flomoxef sodium and levofloxacin $(2.1 \%, \mathrm{p}<0.0001$, Table 4). Furthermore, the incidence rate of acute prostatitis in 10-core-biopsied patients who were administered flomoxef sodium and levofloxacin was $0.4 \%$, which was significantly lower than that in 10-core-biopsied patients who were administered levofloxacin alone $(4.6 \%, \mathrm{p}<0.0001)$. Logistic regression analysis revealed that the administration of flomoxef sodium was only independently associated with the incidence of overall complications (Table 5).

\section{Discussion}

The present study demonstrated that the additional 4-core biopsies of the mid-peripheral region of the peripheral zone to systematic lateral-peripheral sextant biopsy significantly increased the cancer detection rate compared with sextant biopsy alone, es- pecially in a subgroup of patients with a PSA of 4.0 $10.0 \mathrm{ng} / \mathrm{ml}$, without increasing the rate of clinically insignificant cancer. Moreover, the incidence rate of acute prostatitis in 10-core-biopsied patients who were administered flomoxef sodium and levofloxacin was significantly lower than in those who were administered levofloxacin alone. As a result, the overall complication rate was also significantly reduced in 10-core -biopsied patients who were administered flomoxef sodium and levofloxacin.

The systematic sextant biopsy method is a 'gold standard' technique and involves biopsy of the prostate gland in the mid-peripheral region ${ }^{2}$. Terris et al. performed transrectal ultrasound with mid-peripheral sextant and lateral-peripheral biopsies, and reported that laterally positioned systematic sextant biopsies improve the diagnosis rate ${ }^{5}$. Accordingly, we adopted the lateral-peripheral sextant biopsy proposed by Terris et al. as our standard sextant biopsy protocol. They also reported that in $7.3 \%$ of patients cancer was detected solely in the mid-peripheral region.

Table 3. Number of Prostate Cancer Patients Detected by 10-Core Biopsy According to Cancer Detected Region

\begin{tabular}{cccccc}
\hline & Total N & $\begin{array}{c}\text { PCA patients with cancer } \\
\text { in mid-peripheral region only }\end{array}$ & $\begin{array}{c}\text { PCA patients with cancer } \\
\text { in lateral-peripheral region only }\end{array}$ & $\begin{array}{c}\text { PCA patients with cancer } \\
\text { detected in both regions }\end{array}$ \\
\hline \multirow{6}{*}{ PSA (ng/ml) } & total & 659 & $56(8.5 \%)$ & $150(22.8 \%)$ & $453(68.7 \%)$ \\
& $4.0-10.0$ & 289 & $36(12.5 \%)$ & $92(31.8 \%)$ & $161(55.7 \%)$ \\
& $10.1-20.0$ & 163 & $13(8.0 \%)$ & $38(23.3 \%)$ & $112(68.7 \%)$ \\
\hline \multirow{2}{*}{ PSAD } & $>20.0$ & 207 & $7(3.4 \%)$ & $20(9.7 \%)$ & $180(87.0 \%)$ \\
\hline \multirow{2}{*}{ PV $(\mathrm{ml})$} & $<0.2$ & 91 & $13(14.3 \%)$ & $37(40.7 \%)$ & $41(45.1 \%)$ \\
& $\geq 0.2$ & 472 & $36(7.6 \%)$ & $92(19.5 \%)$ & $344(72.9 \%)$ \\
\hline
\end{tabular}

$\mathrm{N}$, number; PCA, prostate cancer; PSA, prostate specific antigen; PSAD, PSA density; PV, prostate volume

Table 4. Complication Rate among Biopsied Patients Administered with or without Flomoxef Sodium

\begin{tabular}{|c|c|c|c|c|c|}
\hline \multirow[b]{2}{*}{ Complication } & \multicolumn{3}{|c|}{ Biopsy groups } & \multicolumn{2}{|c|}{$\mathrm{p}$ value } \\
\hline & $\begin{array}{c}\text { Sextant biopsy group }{ }^{\dagger} \\
(\mathrm{n}=251)\end{array}$ & $\begin{array}{c}\text { 10-core biopsy group } \\
\text { without FMOX } \\
(\mathrm{n}=531)\end{array}$ & $\begin{array}{c}\text { 10-core biopsy group } \\
\text { with FMOX } \\
(\mathrm{n}=749)\end{array}$ & $\dagger \mathrm{vs}+$ & $\$ \mathrm{vs} \S$ \\
\hline Prostatitis & $8(3.1 \%)$ & $26(4.6 \%)$ & $2(0.4 \%)$ & 0.2782 & $<0.0001$ \\
\hline Vasovagal response & $0(0 \%)$ & $7(1.3 \%)$ & $7(0.9 \%)$ & 0.0682 & 0.5103 \\
\hline Urinary retention & $0(0 \%)$ & $5(0.9 \%)$ & $4(0.5 \%)$ & 0.1237 & 0.3863 \\
\hline Bleeding & $1(0.4 \%)$ & $5(0.9 \%)$ & $3(0.4 \%)$ & 0.4185 & 0.2238 \\
\hline Epididymitis & $0(0 \%)$ & $1(0.2 \%)$ & $0(0 \%)$ & 0.5337 & 0.3863 \\
\hline Overall & $9(3.6 \%)$ & $44(8.8 \%)$ & $16(2.1 \%)$ & 0.0099 & $<0.0001$ \\
\hline
\end{tabular}

$\dagger$ Sextant biopsied group, those were administered with levofloxacin alone

$\$ 10$-core biopsy group without FMOX, those were administered with levofloxacin alone

$\S 10$-core biopsy group with FMOX, those were administered with flomoxef sodium in addition to levofloxacin

FMOX, flomoxef sodium 
Table 5. Logistic Regression Analysis for Identifying the Indicators Associated with the Occurrence of Overall Complication

\begin{tabular}{lccc}
\hline Variable & Odds ratio & $95 \%$ CI & p value \\
\hline Age, $<70$ vs $\geq 70$ & 1.249 & $0.778-2.005$ & 0.3577 \\
PSA, $<10$ vs $\geq 10$ & 1.073 & $0.664-1.734$ & 0.773 \\
PV,$<20$ vs $\geq 20$ & 0.515 & $0.219-1.211$ & 0.1282 \\
PSAD, $<0.2$ vs $\geq 0.2$ & 1.291 & $0.758-2.203$ & 0.3468 \\
Biopsy, sextant vs 10-core & 0.724 & $0.355-1.475$ & 0.3734 \\
FMOX administered, no vs yes & 3.547 & $2.014-6.247$ & $<0.0001$ \\
\hline
\end{tabular}

CI, confidence interval; PSA, prostate specific antigen; PV, prostate volume; PSAD, PSA density; FMOX, flomoxef sodium

Thus, we planned to add 4-core biopsies obtained from the base and mid gland in the mid-peripheral region bilaterally. Thereafter, the majority of biopsy methods proposed focus on the benefit of additional lateral biopsies ${ }^{11,12)}$ and reported that the midline portion of the biopsy contributes no additional advantage with respect to cancer detection according to the data of Western countries ${ }^{13}$. However, our data demonstrate that additional 4-core biopsies of the mid-peripheral region significantly improved the cancer detection rate and $12.5 \%$ of the cancer was detected in only mid-peripheral region in the patients with PSA grey zone $(4.0-10.0 \mathrm{ng} / \mathrm{ml}$; $\mathrm{p}<0.05)$. Studies using prostatectomy specimens have reported a racial difference in tumor location within the prostate ${ }^{14,15)}$, and Inaba et al. reported that prostate cancer in Japanese men was characterized by a high frequency of midline portion and anterior cancer ${ }^{16}$. In addition, Soga et al. reported that midline additional biopsies are associated with improved accuracy regarding cancer detection in Japanese patients ${ }^{17)}$, which is in agreement with our data. Thus, the alternative systematic prostate biopsy which includes additional midline biopsies may be necessary for Japanese patients.

Although there is a growing interest in imaging studies, magnetic resonance imaging (MRI)-targeted techniques cannot be recommended for routine initial biopsy until issues related to the quality of imaging, targeting strategies, and cost are fully resolved ${ }^{18)}$. Furthermore, previous studies have suggested that MRI-targeted biopsy should be performed with concurrent systematic biopsy because clinically significant cancer can be missed by targeted biopsies alone ${ }^{19-21)}$. These reports suggested that systematic biopsy still has an important role in the era of MRI-target prostate biopsy ${ }^{22)}$.

In 2002, Griffith et al. reported that the use of levofloxacin before transrectal prostate biopsy is effective to reduce symptomatic urinary tract infection, especially in low-risk patients ${ }^{23)}$. Thus, we initially started routine prophylaxis consisting of oral levofloxacin at our hospital. Albeit with using levofloxacin, the rate of prostatitis was up to $4.6 \%$ in the 10 core biopsy group. Concurrently, fluoroquinoloneresistant infectious complications were reported to be increased according to a Japanese cohort ${ }^{24,25)}$ and fluoroquinolone-resistant acute prostatitis was also observed in patients at our hospital. The susceptibility of flomoxef sodium to fluoroquinolone-resistant E. coli at our hospital was $98 \%$ at that time based on the data from the infectious control unit of our hospital. Thus, a single-dose intravenous injection of flomoxef sodium has been administered immediately before the procedure since April 2009 for all patients. As a result, the rate of acute prostatitis in 10-core -biopsied patients who were administered flomoxef sodium and levofloxacin was significantly lower than in those who were administered levofloxacin alone $(\mathrm{P}<0.0001)$. After administering flomoxef sodium, acute prostatitis only developed in 2 patients. Fluoroquinolone-resistant E. coli was not detected thereafter.

Our prophylaxis method is different from that recommended in the Japanese guidelines ${ }^{6}$, but our original method prevented acute prostatitis. The infection control unit of our hospital updated the data of susceptibility testing in 2019, and the susceptibility of E. coli to flomoxef sodium, including fluoroquinolone-resistant E. coli, is $98 \%$ and that of ESBL E. coli is $98 \%$; therefore, the rate of susceptibility has not decreased since 2009. In general, the frequency of drug-resistant bacteria and the rate of drug susceptibility varies across countries, regions, and races $^{26}$. We believe that the regimen for prophylactic 
antibiotic administration can be altered based on susceptibility testing at each hospital.

The present study has some limitations. It was retrospective in nature, and data were retrospectively collected using electronic medical records and some data were lacking (e.g., blood culture testing, and PV in some patients). In particular blood culture testing was not performed for all of the patients who developed acute prostatitis, thus detailed data were lacking. The PV was significantly higher in the sextant biopsy group than in the 10-core biopsy group, which may have influenced the cancer detection rate.

\section{Conclusions}

The present study demonstrated that the additional 4-core biopsies of the mid-peripheral region to systemic lateral-peripheral sextant biopsy significantly increased the cancer detection rate compared with sextant biopsy alone; this was particularly true, in the subgroup of patients with a PSA of 4.0 $10.0 \mathrm{ng} / \mathrm{ml}$, without increasing the rate of clinically insignificant cancer. The incidence rate of acute prostatitis was increased in the patients with 10-core biopsy; however, it was significantly decreased after the use of flomoxef sodium in addition to levofloxacin.

\section{Conflicts of Interest}

The authors have nothing to disclose.

\section{References}

1) Kakehi Y, Sugimoto M, Taoka R. Evidencedbased clinical practice guideline for prostate cancer (summary: Japanese Urological Association, 2016 edition). Int J Urol 2017; 24: 648-666.

2) Hodge KK, McNeal JE, Terris MK, et al. Random systematic versus directed ultrasound guided transrectal core biopsies of the prostate. J Urol 1989; 142: 71-74.

3) Eskew LA, Bare RL, McCullough DL, et al. Systematic 5 region prostate biopsy is superior to sextant method for diagnosing carcinoma of the prostate. J Urol 1997; 157: 199-202.

4) Levine MA, Ittman M, Melamed J, et al. Two consecutive sets of transrectal ultrasound guided sextant biopsies of the prostate for the detection of prostate cancer. J Urol 1998; 159: 471-475.

5) Terris MK, Wallen EM, Stamey TA. Comparison of mid-lobe versus lateral systematic sextant biopsies in the detection of prostate cancer. Urol Int 1997; 59: 239-242.

6) Yamamoto $S$, Shigemura K, Kiyota H, et al. Jap- anese Research Group for UTI. Essential Japanese guidelines for the prevention of perioperative infections in the urological field: 2015 edition. Int J Urol 2016; 23: 814-824.

7) Loeb S, Vellekoop A, Ahmed HU, et al. Systematic review of complications of prostate biopsy. Eur Urol 2013; 64: 876-892.

8) Donzella JG, Merrick GS, Lindert DJ, et al. Epididymitis after transrectal ultrasound-guided needle biopsy of prostate gland. Urology 2004; 63: 306-308.

9) Ergani B, Çetin T, Yalçın MY, et al. Effect of rectal mucosa cleansing on acute prostatitis during prostate biopsy: A randomized prospective study. Turk J Urol 2020; 46: 159-164.

10) Levy MM, Fink MP, Marshall JC, et al. 2001 SCCM/ESICM/ACCP/ATS/SIS International Sepsis Definitions Conference. Crit Care Med 2003; 31: 1250-1256.

11) Presti JC, Chang JJ, Bhargava V, et al. The optimal systematic prostate biopsy scheme should include 8 rather than 6 biopsies: results of a prospective clinical trial. J Urol 2000; 163: 163 166.

12) Durkan GC, Sheikh N, Johnson P, et al. Improving prostate cancer detection with an extendedcore transrectal ultrasonography-guided prostate biopsy protocol. BJU Int 2002; 89: 33-39.

13) Eichler K, Hempel S, Wilby J, et al. Diagnostic value of systematic biopsy methods in the investigation of prostate cancer: a systematic review. J Urol 2006; 175: 1605-1612.

14) Takahashi H, Epstein JI, Wakui S, et al. Differences in prostate cancer grade, stage, and location in radical prostatectomy specimens from United States and Japan. Prostate 2014; 74: 321-325.

15) Hashine K, Ueno Y, Shinomori K, et al. Correlation between cancer location and oncological outcome after radical prostatectomy. Int J Urol 2012; 19: 855-860.

16) Inaba $H$, Kimura $T$, Onuma $H$, et al. Tumor Location and Pathological Features of Latent and Incidental Prostate Cancer in Contemporary Japanese Men. J Urol 2020; 204: 267-272.

17) Soga N, Yatabe Y, Ogura Y, et al. Additional Midline Biopsies of the Peripheral Zone Associated with the First Endorectal Standard Sextant Pattern Improves the Accuracy of Prostate Cancer Detection in Japanese Patients. Curr Urol 2015; 8: 74-78. 
18) Fulgham PF, Rukstalis DB, Turkbey IB, et al. AUA Policy Statement on the Use of Multiparametric Magnetic Resonance Imaging in the Diagnosis, Staging and Management of Prostate Cancer. J Urol 2017; 198: 832-838.

19) Mannaerts CK, Kajtazovic A, Lodeizen OAP, et al. The added value of systematic biopsy in men with suspicion of prostate cancer undergoing multiparametric MRI-targeted biopsy. Urol Oncol 2019; 37: 298.e1-298. e9.

20) Elkhoury FF, Felker ER, Kwan L, et al. Comparison of Targeted vs Systematic Prostate Biopsy in Men Who Are Biopsy Naive: The Prospective Assessment of Image Registration in the Diagnosis of Prostate Cancer (PAIREDCAP) Study. JAMA Surg 2019; 154: 811-818.

21) Filson CP, Natarajan S, Margolis DJ, et al. Prostate cancer detection with magnetic resonanceultrasound fusion biopsy: The role of systematic and targeted biopsies. Cancer 2016; 122: 884892.
22) Sugano D, Sidana A, Calio B, et al. MRI-targeted biopsy: is systematic biopsy obsolete? Can J Urol 2017; 24: 8876-8882.

23) Griffith BC, Morey AF, Ali-Khan MM, et al. Single dose levofloxacin prophylaxis for prostate biopsy in patients at low risk. J Urol 2002; 168: 1021-1023.

24) Shigehara K, Miyagi T, Nakashima $T$, et al. Acute bacterial prostatitis after transrectal prostate needle biopsy: clinical analysis. J Infect Chemother 2008; 14: 40-43.

25) Feliciano J, Teper E, Ferrandino M, et al. The incidence of fluoroquinolone resistant infections after prostate biopsy are fluoroquinolones still effective prophylaxis? J Urol 2008; 179: 952955.

26) Lee JW, Park SC, Kim MK, et al. Prevalence of antimicrobial resistance in normal rectal flora of patients undergoing transrectal ultrasonographyguided prostate biopsy in Korea. Int $\mathrm{J}$ Urol 2014; 21: 811-814. 